\title{
Similarity of Gaze Characteristics of Monozygotic Twins and Triplets
}

\author{
Takahiro Higuchi $^{1}$, Yuko Ishizaki ${ }^{2}$, Atsushi Noritake ${ }^{3}$, Yoshitoki Yanagimoto ${ }^{4}$, \\ Hodaka Kobayashi $^{5}$, Kae Nakamura ${ }^{3}$ and Kazunari Kaneko ${ }^{6}$ \\ ${ }^{1}$ Department of Pediatrics, Kansai Medical University \\ ${ }^{2}$ Department of Developmental Pediatrics Donated by Nabari City, Kansai Medical University \\ ${ }^{3}$ Department of Physiology, Kansai Medical University \\ ${ }^{4}$ Division of Behavioral Development Department of System Neuroscience, \\ ${ }^{5}$ National Institute for Physiological Sciences
}

\begin{abstract}
Twins and triplets often show similar eye-gaze behavior in social scenes (Kennedy et al, 2017). However, it remains unclear what components in social scenes provide such similarity in eyegaze behavior. Here, we hypothesized that joint attention in social scenes may recruit common attention-related neurocircuitry of twins and triplets, and thereby providing similar eye-gaze behavior.

To examine this hypothesis, we measured eye positions of one set of monozygotic twins (two 3year-old females) and one set of monozygotic triplets (three 5-year-old females) with typical development (TD) to that of age-matched children with TD $(\mathrm{n}=25$; median $5 \mathrm{y}, 11$ males and 14 female), while they performed free viewing of several static classroom and scenery images. The classroom images contained joint attention situations, in which a teacher pointed at objects on a screen and students gazed at them, and scenery images contained naturalistic scenes including flowers, trees, and humans but without social interactions. The twins spent similar time gazing at regions of the teacher's face and his pointing objects in the classroom scene while the agematched children showed large variance in gaze time at those regions. In contrast, the similarity in gaze time at specific regions was not observed in the scenery images. These trends were also observed in the triplets. Our results suggest that gaze time of the twins and the triplets at regions related to joint attention in social scenes are heritable.
\end{abstract}

Keywords: twins; triplets; gaze characteristics; similarity; genetic factor 